\title{
Experiments and DEM Simulations of Granular Ratcheting
}

\author{
Gianluca Zorzi ${ }^{1, \star}$, Riccardo Artoni ${ }^{2, \star \star}$, and Fabio Gabrieli ${ }^{3, \star \star \star}$ \\ ${ }^{1}$ GuD CONSULT, Darwinstraße 13, 10589, Berlin, Germany \\ ${ }^{2}$ LUNAM Université, IFSTTAR, MAST, GPEM, F-44340 Bouguenais, France \\ ${ }^{3}$ Icea Dept., University of Padova, Via Ognissanti 39, 35129 Padova, Italy
}

\begin{abstract}
In this work we studied the effect of cyclic loading on a granular packing by means of numerical simulations and experiments. A confined packing of glass beads was prepared and one of the walls was moved cyclically with a prescribed amplitude of the order of the particle diameter. Different amplitudes were tested, and their effect on the free surface evolution, the force transmitted to the moving wall and the displacement patterns in the material was characterized. Discrete numerical simulations were also carried out with the specific purpose of evaluating the effect of the particle shape on the dynamics of the system. The displacement amplitude of the moving wall was shown to increase the maximum force experienced at the end of the compressive phase of the wall movement; the angularity of the particles had a similar effect. Force-wall displacement curves displayed a peculiar hysteretic behavior. The evolution of the system towards an asymptotic state was shown to be faster for spheres than for angular particles; the latter displayed an interesting long-time evolution of the force-displacement paths which deserves deeper investigations.
\end{abstract}

\section{Introduction}

Granular ratcheting is an interesting phenomenon which arises spontaneously when a granular material is subjected to cyclic loads or, more generally, to cyclic strains. It consists in the accumulation of irreversible strains in the granular mass and in a modification of its mechanical response. The effects of granular ratcheting has been shown in the form of convective flow [1,2], densification, soil settlementand a stiffening of the soil-structure interaction (SSI) [3]. This evolution of the soil under cyclic loading is mainly due to the rearrangement of the initial packing and could be an important aspect to be considered for the vulnerability of the underground structures. From an engineering point of view it is important to understand and predict this change of the soil behaviour under cyclic loading especially in the long-term SSI problem. In this paper, in order to characterize the ratcheting phenomenon, joint laboratory tests and DEM simulations of a granular packing subject to the cyclic displacement of a lateral wall were carried out. We focused on the effect of the displacement amplitude and of the particle shape on forces transmitted to the moving wall, and on the macroscopic motion of the granular mass. In the following we first discuss experimental conditions and setup, and then introduce the numerical method.

\footnotetext{
^e-mail: zorzi@gudconsult.de

$\star \star$ e-mail: riccardo.artoni@ifsttar.fr

$\star \star \star$ e-mail: fabio.gabrieli@unipd.it
}

\section{Experiments}

\subsection{Experimental set-up}

Experimental tests were performed using a glass box of size $L=150 \times W=50 \times H=90 \mathrm{~mm}$ in which one of the lateral walls was cyclically moved with a prescribed amplitude (see Fig. 1 for a sketch of the set-up). Glass beads were poured in the box and then leveled to reach a material height of $90 \mathrm{~mm}$.

All tests were conducted in quasi-static conditions with $(d x / d t)_{\max }<10^{-3} \mathrm{~mm} / \mathrm{s}$ by means of a manual inbuilt micrometrical screw, equipped with precision bearings to avoid slack and a load cell in order to record the horizontal lateral force. A triangle wave horizontal displacement was imposed by the screw with different amplitudes. The materials used in the tests discussed here were glass beads (soda-lime silicaglass) with an average diameter of $1.5 \mathrm{~mm}$. Results from other tests (for different materials and particle diameters), and more information regarding the preparation and conduction of the tests can be found in [2]. The present paper will focus on two amplitude/particle diameter ratios (named $A / D$ in the following) equal to 1 and 0.50 . For each $A / D$ value, 50 wall displacement cycles were performed. A digital camera Canon EOS 400D equipped with EF-S 18-55 lens was placed perpendicular to the box in order to capture the grain motions behind the transparent wall. The image sequences were analysed with an image analysis module for MATLAB called GeoPIV_RG [4], which implements image cross-correlation algorithm to identify the motions of a grid of image patches. 
(a)

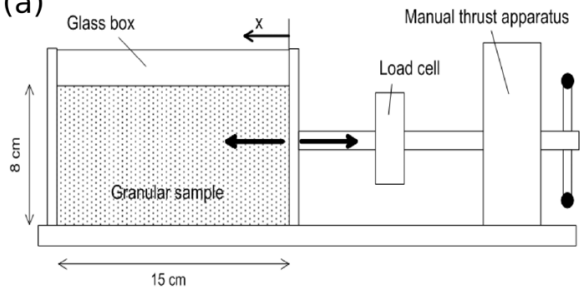

(b)

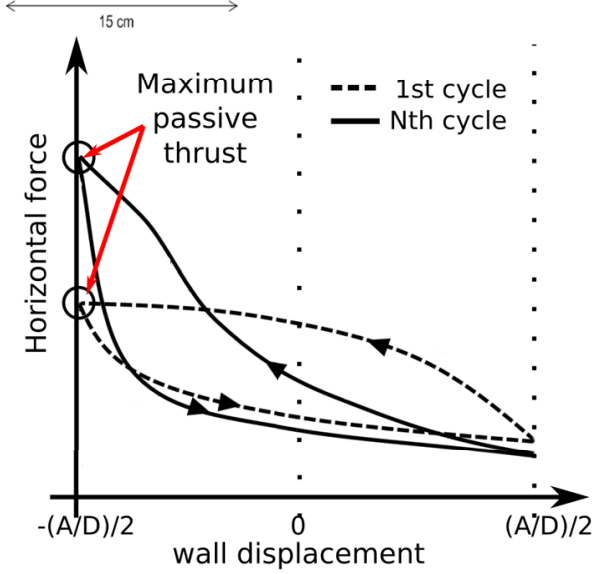

Figure 1. (a) Experimental device. A $L=150 \times W=50 \times H=90$ $\mathrm{mm}$ rectangular cuboid is filled with a granular material (glass beads). One of the lateral walls is cyclically moved with a given amplitude in quasistatic conditions. (b) Typical profiles of the horizontal force exerted on the wall as an function of the wall displacement.

\subsection{Experimental results}

Due to the periodic displacement of the wall, the force exerted by the material on the wall has a typical hysteretic evolution, which is sketched in Fig. 1. During the (passive) compression phase, the force increases progressively with the wall displacement, while in the (active) relaxation phase the system follows another path, characterized by lower values of the force. The hysteresis loop evolves during the cycles. Figure 2 displays the evolution of the maximum passive thrust (i.e. the maximum force exerted during each compression cycle of the wall displacement) with the number of cycles. It is evident that the maximum force increases with the number of cycles, and that an increase in the displacement amplitude yields an increase in the force. It is reasonable to expect that the maximum passive thrust will reach an asymptote for a sufficiently large number of cycles. In our experiments the maximum number of cycles was not sufficient to characterize this long time behavior of the ratcheting phenomenon.

Figure 3 shows the free surface profile after 50 cycles for the two values of A/D introduced above. It is clear that both tests show a settlement of the granular material close to the moving wall. This settlement is only slightly higher the larger the amplitude.

The convective flux induced by the cyclic displacement of the lateral wall can be appreciated by tracking the position of a grid of patches in the sequence of images taken when the screw passes at the zero position. This is shown in Figure 4. It is clear that the larger displace-

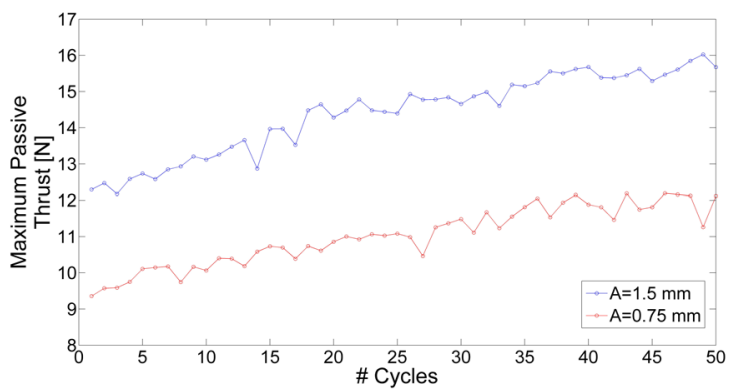

Figure 2. Evolution of the maximum passive thrust (i.e. the maximum force exerted during each compression cycle of the wall displacement) with the number of cycles.

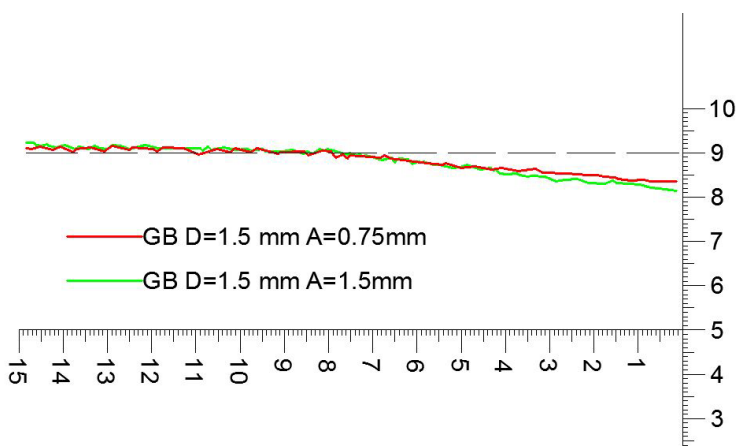

Figure 3. Free surface profiles after 50 cycles, for $1.5 \mathrm{~mm}$ glass beads with $\mathrm{A} / \mathrm{D}=1$ (green line)and 0.50 (red line). The dashed line corresponds to the initial surface level.

ment amplitude displays a larger permanent displacement. Most of irreversible strains are just behind the moving wall where the particles move exhibiting a net vertical displacement, which is not due to a simple gravitational densification, since it persists at each cycle. Deeper particles near the wall deviates from the vertical, and the horizontal component results larger for larger displacement amplitude. In the middle region of the sample the material moves with different magnitude but approximately with the same direction. Far away from the wall the cumulative displacement is gentle and diagonal-upward, compatible with a passive stress condition. No closure of the convective flux (i.e. permanent displacement towards the wall) is detected near the ground surface during the tests. This is very likely consistent with the lack of an asymptotic behaviour in the maximum passive force trend.

\section{Numerical simulations}

\subsection{Numerical method}

Numerical simulations were performed using the non smooth contact dynamics method [5], as implemented in the LMGC90 open source framework [6]. Simulations were carried out in a rectangular cuboid geometry of dimensions $L=30 d \times W=10 d \times H=20 d$, where $d$ is the particle characteristic length. Simulations were performed in dimensionless units with lengths rescaled by $d$, 


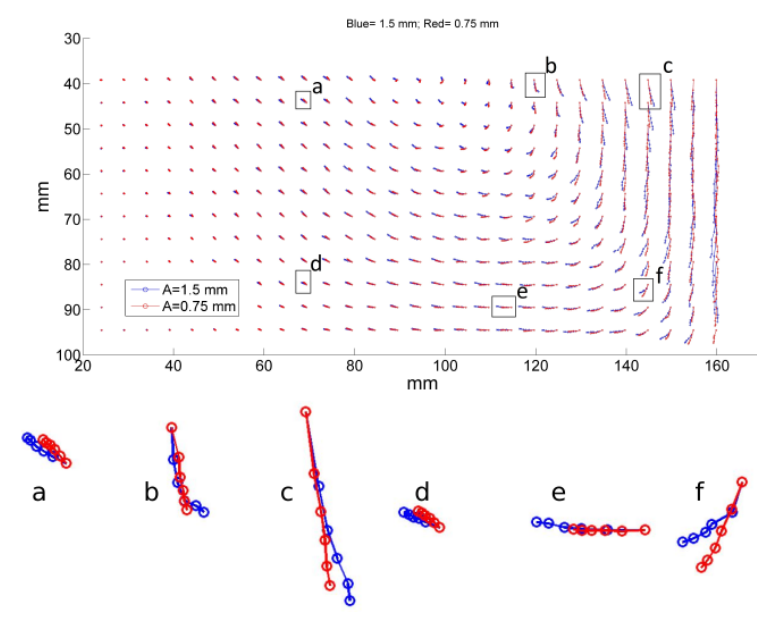

Figure 4. (top) Paths of the irreversible displacements after 50 cycles obtained by crosscorrelation PIV, for two values of the displacement amplitude. (bottom) Focus on some peculiar paths with the letters referring to the top picture.

time rescaled by $\sqrt{d / g}$, masses rescaled by $\rho d^{3}$. In order to study the effect of the the particle shape on the ratcheting phenomenon, two types of monodisperse granular particles with different shapes were used in the simulations (spheres, and pinacoids, as sketched in Fig. 5). The pinacoid is an example of a very angular particle which was already discussed by Camenen et al. [7]. The one chosen in this work has all the three axis equal to $d$ and a side angle of $60^{\circ}$. Contacts were modeled as inelastic with a particle-particle and particle-wall friction coefficient of $\mu_{p}=\mu_{p w}=0.5$. The initial packing was obtained by a rainfall simulation with a stability criteria of total kinetic energy over total potential energy less than $10^{-8}$. Once a stable packing was reached, the ratcheting simulation started. This paper will present two different normalized displacement amplitude $A / D=0.5$ and 1 tested for each particle shape. The same displacement pattern starting from $(A / D) / 2$, inverting the motion and approaching $-(A / D) / 2$ was imposed for 100 cycles. Wall velocity was maintained constant and slow to simulate quasi-static conditions. In order to have approximately the same initial level independently of particle shape, the number of particles was different between spheres $(N=5590)$ and pinacoids $(N=5990)$.

\subsection{Numerical results}

The evolution of the free surface profiles with the number of cycles for different particle shapes and displacement amplitudes is shown in Figure 5. It is clear that the largest part of the evolution occurs between 1 and 50 cycles, and that particle shape strongly influences the final surface shape. Increasing the angularity of particles yields a more pronounced surface variation. Moreover, as it was shown in experiments, increasing the displacement amplitude yields a slightly more pronounced variation of the surface profile. The surface variation is more important than in the experiments; this is probably an effect of

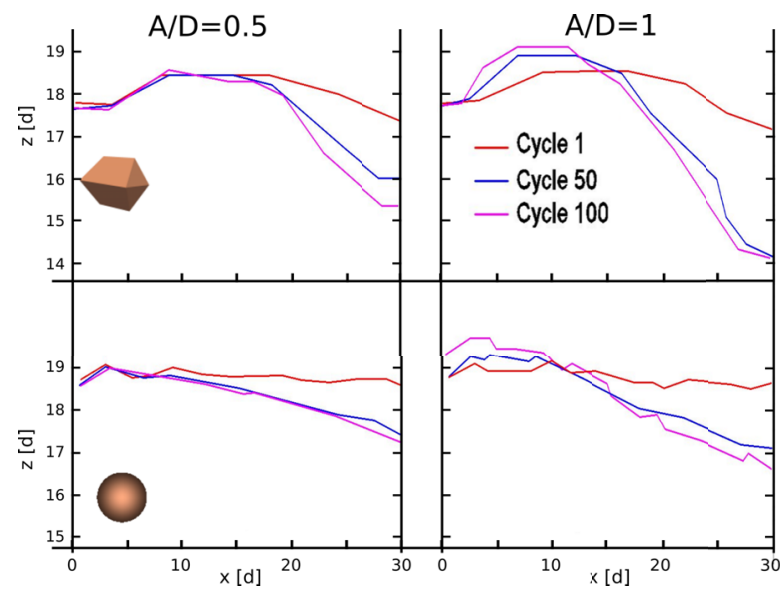

Figure 5. Free surface profiles for different cycle numbers for simulations with different particle shapes and displacement amplitudes.

the difference in the ratio between particle size and system dimension.

In figure $6 a$, the maximum passive thrust obtained from the simulations is plotted as a function of the number of cycles. The force displays strong fluctuations from one cycle to the other, probably due to the relatively small system size in terms of number of particles. However, we can see that an initial increase of the force is followed by a nearly constant value for all the simulations after approximately 50 cycles. This is consistent with the fact that free surface displays only a small evolution after 50 cycles, as it was shown in Figure 5.

The force-wall displacement profiles for spheres (Fig. 6b) confirm this point. Between 50 and 100 cycles, the force-displacement curves follow nearly the same hysteresis loop. The system has reached an asymptotic state. As regards pinacoids (Fig. 6c), however, even if the free surface displays little evolution and the maximum force is relatively constant, the hysteresis loops definitely continue to evolve from 50 to 100 cycles. While displaying nearly the same final value, the force displacement curve during the compressive phase is characterized by an increasingly nonlinear path, with a steeper evolution. The total work needed to move the wall in a compressive cycle seems to decrease. As concerns the effect of displacement amplitude, Fig. 6a shows that the larger the $A / D$ value the larger are the forces, as it was shown by the experimental results. Particle shape also affects the maximum force values, with the angular particles displaying larger values of the force.

\section{Conclusions}

In this work we studied the effect of cyclic loading on a granular packing by means of numerical simulations and experiments. A packing of glass beads confined between four lateral walls was prepared and one of the walls was moved cyclically with a prescribed amplitude of the order of the particle diameter. Different amplitudes were tested, and their effect on the free surface evolution, the 
(a)

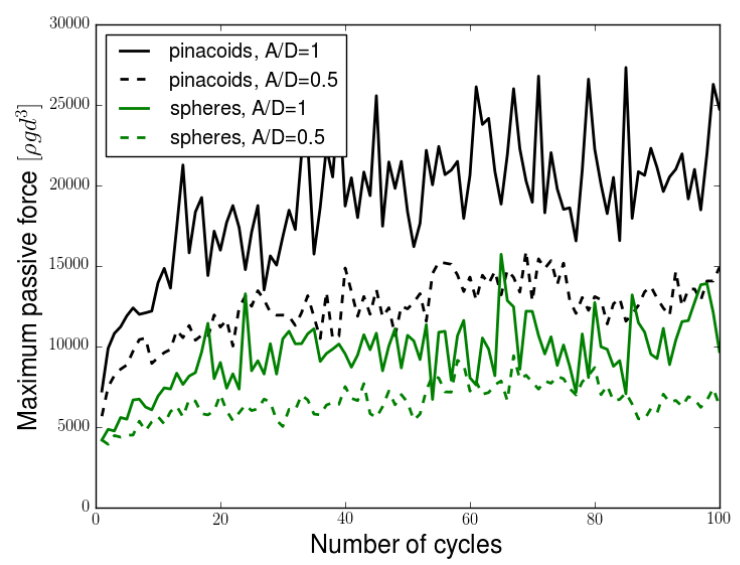

(b)

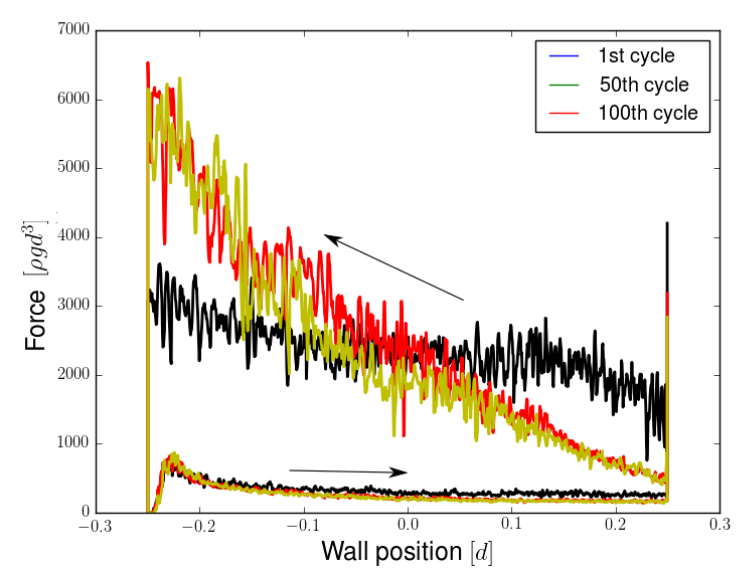

(c)

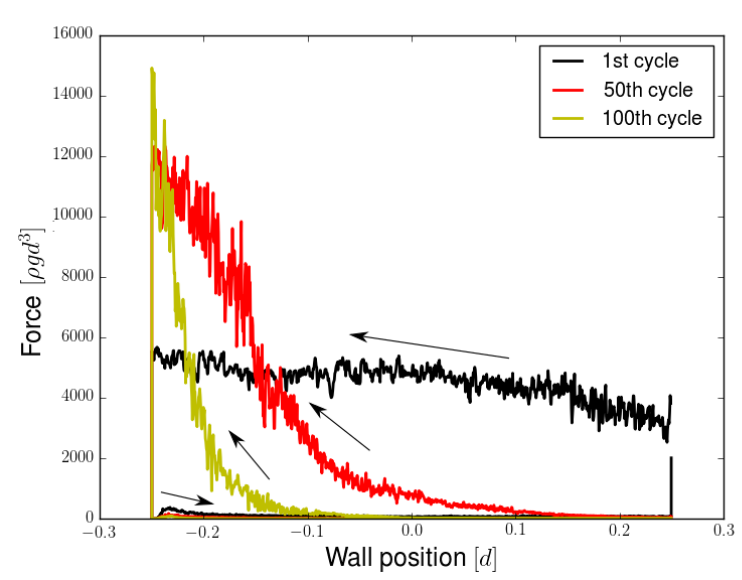

Figure 6. (a) Maximum passive thrust as a function of the number of cycles for spheres, pinacoids and two values of the displacement amplitude $A / D$. (b-c) Hysteresis loops for spheres (b) and pinacoids (c) with $A / D=0.5$ at different cycles force transmitted to the moving wall and the displacement patterns in the material was characterized. Numerical simulations by the nonsmooth contact dynamics method were also carried out with the specific purpose of evaluating the effect of the particle shape (spheres vs angular particles) on the dynamics of the system. The displacement amplitude of the moving wall was shown to increase the maximum force experienced at the end of the compressive phase of the wall movement; the angularity of the particles had the same effect. The evolution of the system towards an asymptotic state was shown to be faster for spheres than for angular particles; the latter displayed an interesting evolution of the force-displacement hysteresis loop which is probably related with microstructural changes and deserves deeper investigations. The effect of system size and other particle shapes will also be addressed in the future.

\section{Acknowledgements}

The numerical simulations were carried out at the CCIPL (Centre de Calcul Intensif des Pays de la Loire) under the project MTEEGD.

\section{References}

[1] F. Alonso-Marroquín, H.J. Herrmann, Phys. Rev. Lett. 92, 054301 (2004)

[2] F. Gabrieli, G. Zorzi, R. Wan, in Geomechanics from Micro to Macro (CRC Press, 2014), pp. 601-606.

[3] P. Cuéllar, S. Georgi, M. Baeßler, W. Rücker, Granular Matter 14, 11 (2012)

[4] D. White, W. Take, M. Bolton, Geotechnique 53, 619 (2003)

[5] M. Jean, Computer Methods in Applied Mechanics and Engineering 177, 235 (1999)

[6] M. Renouf, F. Dubois, P. Alart, Journal of Computational and Applied Mathematics 168, 375 (2004)

[7] J.F. Camenen, Y. Descantes, P. Richard, Phys. Rev. E 86, 061317 (2012) 